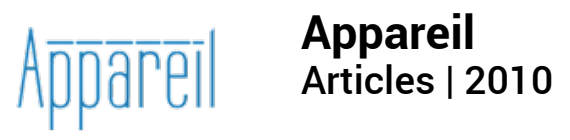

\title{
« Femmes, je vous aime »... derrière une vitrine. elles@centrepompidou.
}

critique de l'exposition elles@centrepompidou

\section{Nathanaël Wadbled}

\section{CpenEdition}

Journals

Édition électronique

URL : http://journals.openedition.org/appareil/1007

DOI : 10.4000/appareil. 1007

ISSN : 2101-0714

Éditeur

MSH Paris Nord

Référence électronique

Nathanaël Wadbled, « « Femmes, je vous aime »... derrière une vitrine.

elles@centrepompidou. », Appareil [En ligne], Articles, mis en ligne le 10 mars 2010, consulté le 30 juillet 2020. URL : http://journals.openedition.org/appareil/1007 ; DOI : https://doi.org/10.4000/appareil. 1007

Ce document a été généré automatiquement le 30 juillet 2020.

\section{(†) $\ominus$

Appareil est mis à disposition selon les termes de la Licence Creative Commons Attribution - Pas d'Utilisation Commerciale - Pas de Modification 4.0 International. 


\title{
« Femmes, je vous aime »... derrière une vitrine. elles@centrepompidou.
}

Critique de l'exposition elles@centrepompidou

\author{
Nathanaël Wadbled
}

\section{Introduction : un rapport désintéressé aux œuvres}

\section{Enfin des femmes artistes !}

1 C'est un cliché, un lieu commun que de partir de l'article de Lindan Nochlin « Pourquoi n'y a-t-il pas de grands artistes femmes? $»^{1}$ pour commenter ou commencer une réflexion sur l'exposition des œuvres artistiques des femmes. Un grand nombre de catalogues d'expositions et d'ouvrages sur le sujet s'y sont essayés et se sont placés sous ce haut patronage.elles@centrepompidou ne fait pas exception, et un commentaire de l'exposition aurait du mal lui aussi à s'y soustraire.

2 L'enjeu affiché de elles est de répondre à la question de Lindan Nochlin par l'affirmative. Il y aurait désormais, à partir de elles, de grandes femmes artistes dans la mesure où leurs œuvres seraient enfin reconnues comme des œuvres d'art comme les autres. Les œuvres des femmes auraient enfin, après avoir été reléguées à une place mineure, des conditions d'exposition propices à la révélation de leurs qualités esthétiques. La reconnaissance des femmes comme grandes artistes passerait alors par la possibilité de goûter leurs œuvres comme étant simplement des œuvres d'art comme les autres. Le concept explicite de l'exposition est en effet que le genre de l'artiste apparaisse comme non pertinent dans le rapport aux œuvres. Elles est en effet fondée sur le désintérêt pour les conditions contextuelles des œuvres dans lesquelles elles ont pu avoir un rôle social - soit actif d'organisation ou de transformation des perceptions et de la manière de voir les choses soit de révélateur d'une certaine "vision du monde »- comme si dans l'œuvre ne comptait essentiellement que ce qui y est d'art au delà de tout contexte 
plutôt que ce qui y est utilitaire. Le visiteur est dans la même situation que Picasso devant les statues païennes. Il ne voit pas des outils mais des statues, des photographies, des peintures, des films, des sculptures :

«De temps en temps je pense, il y a eu un Petit Bonhomme des Cyclades. Il a voulu faire cette sculpture épatante comme ça. Il croyait faire la Grande Déesse, je ne sais quoi. Il a fait ça. Et moi à Paris, je sais ce qu'il a voulu faire : pas le Dieu, la sculpture. Il ne reste rien de sa vie, rien de ses espèces de Dieu, rien de rien. Rien. Mais il reste ça, parce qu'il a voulu faire une sculpture $»^{2}$.

3 Toute prise en considération du contexte de production des œuvres, même de cet élément minimal qu'est le genre de l'artiste, est exclue. Le principe de sélection de elles@centrepompidou, selon lequel seules des femmes sont exposées, se veut en effet paradoxalement non pertinent dans la mesure où, comme l'annonce le catalogue dès la préface, il ne s'agit pas de mettre en avant un art féminin, ni de produire un objet féministe, mais de montrer qu'en exposant que des femmes, on peut représenter l'histoire de l'art du $\mathrm{xx}^{\mathrm{e}}$ siècle. Les autres éléments du contexte de création des œuvres ne sont pas plus indiqués. Le visiteur de l'exposition n'est ainsi pas devant des œuvres qu'il pourrait replacer dans un certain contexte social ou culturel afin de déterminer l'influence ou l'importance qu'elles ont eues sur les représentations ou la perception par leurs contemporains de ce qu'elles exposent. Peu importe le contexte de leur création, c'est à dire le rôle social et culturel que les œuvres ont pu avoir. Il s'agit d'être simplement devant des œuvres, dont au final il importe peu qu'elles aient servi à infléchir, à bousculer ou à conforter le regard porté sur les conditions de leur production - par exemple le fait qu'il s'agisse de l'œuvre de femmes avec ce que cela implique comme déterminations sociales et culturelles - ou le sujet traité - par exemple le regard particulier des femmes sur certaines choses ou certains genres artistiques. En fait, peu importe que ces œuvres aient eu ou non une destination utilitaire, c'est-à-dire une utilité sociale ou culturelle. Cette suspension n'est cependant pas une perte dans la mesure où elle permet le rapport esthétique à l'œuvre.

\section{Le jugement esthétique des visiteurs}

4 L'exposition délocalise et met sur le même plan des œuvres ayant eu une certaine importance dans le combat féministe, et d'autres n'ayant jamais eu aucune utilité ni utilisation sociale ou culturelle. Le musée leur offre un site commun d'exposition. Par exemple, à côté de Hanna Wilke, Betty Tompkins exclut explicitement tout enjeu politique de la création des Fuck Painting. Au milieu de l'ensemble Eccentric Abstraction (Marthe Wéry, Peintur Venise 82 ou Monique Frydman, Rouge cadrée, Vera Molnar, Quatre éléments distribués, Lee Bontecou, Untitled ou Silvia Bächli, Sans titre XIX, etc.) qui présente des œuvres se réduisant à un travail sur la couleur et les formes, est présenté Big Pink Diagonal/ Big Angie - RFGA de Ghada Amer qui reproduit et interroge la soumission de la femme en cousant sa propre image déformée sans aucune visée esthétisante ni formelle.

Devant une telle collection, le visiteur est amené à ne considérer ces œuvres que séparées de leur utilité féministe. Le visiteur de l'exposition n'est pas devant des témoignages d'une époque de la culture ou de la société. En ce sens, elles n'est pas le lieu de mémoire d'un patrimoine féminin restituant les difficultés et les enjeux propres de la situation ou de la représentation des femmes. L'enjeu de l'exposition, tel que l'explicite le catalogue, peut être effectif: peu importe qu'il s'agisse de femmes 
engagées dans des relations particulières avec leur contexte social. C'est dans cette perspective que, si la présentation de la commissaire de l'exposition affirme que l'un des enjeux de l'exposition est de montrer qu'il est possible de réécrire l'histoire de l'art avec les femmes', elles n'écrit pas une histoire de l'art, c'est-à-dire une recontextualisation et une compréhension des évolutions et influences socioculturelles réciproques des œuvres et de leur contexte. Le catalogue insiste sur ce choix d'exposition présentant les œuvres comme au-delà de leur fonction dans leur contexte.

«Il nous semblait que réduire la création artistique récente à ces attitudes militantes (en référence par exemple à l'exposition "Global Feminism") ne rendait compte que partiellement de la présence croissante et de l'impact des artistes femmes dans l'art contemporain. $»^{4}$

6 elles n'est pas là pour donner un cours d'histoire, mais pour procurer un plaisir esthétique, qui est, pour Kant, inséparable du dépouillement de l'objet de toute destination utilitaire. L'œuvre n'est pas quelque chose d'utile au sens où elle serait le support de représentations ou permettrait leur transformation, comme le serait par exemple un objet religieux où politique. Un tel objet concerne au plus haut point celui qui est devant lui : il sert de support à sa croyance et de référent à sa vie sociale, culturelle et politique. Hors du contexte, cet objet serait privé de cette utilité, il ne serait pas reconnu et utilisé, c'est-à-dire vécu et inclus dans la vie de celui qui le perçoit comme objet ayant cette utilité. Au contraire, les œuvres présentées dans elles, de par le refus de leur mise en contexte, sont séparées de toute utilité possible. L'œuvre est perçue sans la représentation d'une fin et peut alors être considérée comme belle dans sa pure matérialité, plutôt que comme utile en fonction du sens d'une destination. Celle-ci a peut-être existé, ou non, mais c'est sans importance. Pour reprendre une expression kantienne, le jugement alors porté sur les œuvres est désintéressé.

7 Le visiteur ne se sent donc pas concerné par les œuvres qu'il voit : elles n'agissent pas sur lui, n'agissent pas sur ses représentations des femmes. Elles ne servent à rien et n'ont aucune utilité pour le spectateur, autre que de répondre à son goût pour les belles choses. Selon la perspective prise, ce choix d'exposition peut mener à deux jugements antagonistes sur l'exposition. Que les œuvres ne soient pas présentées comme objet du féminisme, mais comme, ainsi que l'affirme le catalogue de l'exposition, des œuvres d'art comme n'importe lesquelles peut être perçu de manière positive ou négative. Si l'intérêt de l'exposition est de faire une belle exposition de belles œuvres comme les autres, alors le visiteur de l'exposition est comblé par ce qu'il voit. Si son intérêt est au contraire de transformer son rapport aux femmes, alors il peut légitimement être déçu. Il s'agit à la fois d'affirmer la place des femmes dans les musées et de faire de leurs œuvres des pièces de musée. Ces œuvres sont ainsi goûtées, mises à disposition du public, mais derrière une double vitrine. Elle est d'exposition, et donne son cadre à l'œuvre d'art, mais aussi de sécurité, et protège de l'influence directe de ce qui se trouve derrière.

8 Cette double logique est d'une certaine manière explicitée par Jean-Louis Déotte, dans son effort pour comprendre le sens et la nature de la prise en charge muséale des objets. Il s'agira d'en suivre les deux versants, l'un après l'autre, pour voir dans quelle mesure ils impliquent des jugements différents en fonction d'intérêts différents: esthétique ou historique. À chaque fois en effet, un intérêt différent oriente l'analyse et légifère, c'est-à-dire reprend les mêmes éléments (œuvres, choix d'exposition, etc.) pour les faire signifier, les ordonner et les intégrer dans une certaine perspective. Ainsi, ce qui sera la marque de la réussite de l'exposition d'un point de vue esthétique pourra 
être celle de son échec d'un point de vue historique. La difficulté, au final, est que l'exposition affirme à la fois une prétention purement esthétique et une prétention féministe dont l'articulation peut définir une certaine époque culturelle avec ses propres intérêts supérieurs, structurant la manière dont les intérêts esthétiques et culturels sont vécus.

\section{Exposer les belles œuvres des femmes}

\section{La belle collection du Musée imaginaire}

9 Les œuvres sont réduites à leur matière, c'est-à-dire à leur pure présence apparaissant au visiteur sans relation avec leur inclusion et leur fonction dans le contexte de leur emploi. Jean-Louis Déotte voit, dans cette suspension du sens que pourrait avoir l'œuvre au profit de la seule perception de sa matière, le principe même de l'exposition d'art. Ce qui est exposé, c'est une œuvre qui n'est qu'une œuvre d'art enfin perceptible comme telle à présent que son utilité est suspendue. Ce qui est devant nous, c'est une composition de formes, de couleurs, de textures, de mouvements, pas un signifiant prenant sens dans un contexte défini où il aurait une certaine fonction intégrée. C'est ce qui, pour Kant, permet d'avoir un rapport esthétique aux œuvres, c'est-à-dire de les trouver belles plutôt qu'utiles. C'est la pure matière qui est alors goûtée.

« Les objets du jugement de goût survivent à ce qu'ils ont été (des finalités), au-delà

(ou en deçà) leur destination primitive, comme matière. Leur au-delà, c'est leur matière, qui est aussi leur retour, dans une dégradation véridique. Ainsi la matière goûtée peut-elle être dite l'au-delà essentiel de l'objet. »

Comme l'indique la préface du catalogue, Il s'agit de stimuler l'imagination par des mises en relation traversant les collections du musée, plutôt que d'écrire la chronologie contextualisée de l'histoire de l'art. Sont ainsi mises ensemble des œuvres disparates de femmes ayant eu des visées et des expériences différentes, dont les postérités sociales, politiques ou culturelles sont différentes. Elles peuvent être présentées ensemble, réunies dans la même expérience de l'exposition car leurs expériences respectives ne sont pas considérées comme intéressant le visiteur. Dans ce rapport, elles sont réunies au delà de leurs utilités incommensurables - d'où l'inutilité de resituer leur destination féministe.

11 En l'occurrence l'organisation de l'exposition en diverses parties réunit les œuvres selon des principes divers sans cohérence générale. «Eccentric Abstraction » est une catégorie de l'histoire de l'art pour décrire un certain mouvement artistique, «Elles @ design » un genre artistique, "Immaterielles » renvoie à une pure recherche formelle et technique. Même si "Corps slogan ", "Feu à volonté ", "Une chambre à soi » peuvent suggérer une certaine thématique féministe sans pour autant insister sur une problématique sociale. Les différentes parties de l'exposition apparaissent comme des suggestions faites au visiteur dans son activité de mise en correspondance de groupes d'œuvres qui lui sont donnés à voir.

Au delà de leurs contextes de création divers et incommensurables ${ }^{5}$, ces œuvres pouvaient entrer en correspondances. C'est ce que Malraux a appelé le Musée imaginaire. Elles met ainsi en relation des œuvres différentes, appartenant à des courants nettement séparés de l'histoire de l'art, à des systèmes de représentation et des idéologies différentes, mais dont les formes, les textures ou les couleurs, en un mot leur 
pure matière, peuvent se répondre, sont susceptibles de correspondances. Il ne s'agit pas de comparaison ou de relation d'influence. L'enchaînement des œuvres se fait par affinités de leur matière. Dans elles, les œuvres s'enchaînent sans autre lien que leurs rapports harmonieux. Devant chaque élément de l'exposition, ce n'est pas à son contexte d'origine ni à son utilité passée ou présente que songe le visiteur; il voit sa matière qu'il associe à d'autres objets, sans qu'il n'y ait de logique dans cette association. Jean Louis Déotte énonce le principe de cette somnolence en citant George Salle :

«En éclairant le fond obscur de notre miroir intérieur, j’y découvrirai bien (dans un bronze chinois) quelques frères chinois de mon bronze. J'y apercevrai aussi de tout autres images: le marbre brisé d'une acanthe épineuse, le bois guilloché d'un fauteuil Louis XVI ou même la face rocailleuse et creusée d'un vieillard de Rembrandt. La sensation présente s'est accrue et vérifiée par l'écho de ses résonances plastiques. Qu'ai-je fait en évoquant ce marbre antique, ce bois français, cette peinture hollandaise ? J'ai amorcé une collection. J'ai spontanément groupé des objets qui, disparates, sont cohérents par leur conformité entre eux, et avec notre œil. J'ai réagi à leur aspect nu. $»^{6}$

13 C'est ce principe qui fait que le visiteur est devant une belle exposition. En effet, les œuvres présentées s'offrent à ce jeu qui remet en jeu, ou plutôt donne du jeu aux œuvres. Il ne s'agit pas seulement de permettre le jugement de goût au sens kantien, mais également de générer ces correspondances inattendues entre des œuvres qui se rencontrent. Elle présente une certaine possibilité d'ordonnancement du Musée imaginaire. Le visiteur qui y déambule le transforme et le reproduit au hasard de la rencontre des œuvres. Cette métamorphose se reproduit dans le regard du visiteur. La rencontre entre les spectateurs et ce qui est accroché fait que ces œuvres ne sont plus des archives mais des occasions de dialogues imprévisibles et créateurs. L'accrochage incite à créer un nouveau dialogue et de nouveaux réseaux sensibles. Chaque élément interagit avec les autres pour former une belle collection, un ensemble que nous goûtons comme esthétique. L'enchaînement laisse se produire une œuvre d'art nouvelle : l'exposition qui est un agencement d'œuvres et d'ensembles d'œuvres. Ainsi les regroupements d'œuvres dans des ensembles thématiques à l'intérieur de l'exposition, obéissent au même principe que l'agencement des œuvres à l'intérieur de chaque ensemble. La capacité à générer de telles rencontres, à la fois entre les œuvres et entre les salles de l'exposition est, dans cette perspective, le talent permettant de considérer la réussite d'une exposition. Elles serait ainsi une belle composition d'œuvres. Cette composition est l'exposition. Celle-ci peut être donc considérée comme une belle composition de formes qui justifie que Kant compte la galerie de tableaux parmi les œuvres d'art. En ce sens, elles peut être en elle-même considérée comme une œuvre d'art, comme toute muséographie réussie.

\section{elles, nom propre de l'exposition}

14 L'organisation de l'exposition et les correspondances qu'elle crée entre les œuvres, que les visiteurs sont amenés à reconstituer à pour nom propre elles. L'art des femmes est le nom propre de cette exposition, de ce musée imaginaire : elles. La question se pose alors de savoir ce qu'est l'art féminin pour le visiteur, c'est à dire à quoi correspond le nom propre de l'exposition pour celui qui la visite et crée de nouvelles correspondances entre les œuvres en fonction de son goût. Celles-ci composent son propre Musée imaginaire à partir de ce que l'exposition lui offre comme matériaux et lui propose 
comme principe de coordination. Ce matériaux, se sont les œuvres présentées, ce principe est le nom propre de l'exposition qui en est comme le signifiant. Cela ne veut pas dire que tous les visiteurs voient la même chose. Chacun a son propre Musée imaginaire, qui est une variation, une possibilité permise par l'exposition. À l'origine, les matériaux lui servent de support, après coup le nom de l'exposition lui sert de signifiant ou de nom propre.

Il n'y a pas de principe déterminant les correspondances qu'il établira dans l'exposition ni les œuvres qu'il en retiendra. Les matériaux proposés sont mis à son entière disposition et il peut les réagencer selon son goût. À l'origine de la visite, il y a cette disponibilité fondamentale. Cette collection est ce qu'aura été l'exposition elles pour lui. Sortant de l'exposition, le visiteur subsumera ce qu'il en retiendra sous le nom proposé. D'une certaine manière donc, la collection originale qu'il se sera constitué recevra ce nom propre a posteriori. L'art des femmes n'est donc pas perçu comme le principe organisateur de l'exposition, mais comme ce qui apparaît rétrospectivement comme étant le principe coordinateur des correspondances aperçues. C'est en ce sens seulement que l'art des femmes en est le seul principe, le principe de coordination du Musée imaginaire. Une fois constitué, le visiteur se souvient d'une certaine manière qu'il était dans une exposition des œuvres d'artistes femmes et donne ainsi sens à sa propre collection. La circulation dans le divers de séries hétérogènes est identifié à ce principe qui pourtant n'a pas présidé a priori à son organisation, mais y est reconnu.

En ce sens, l'art des femmes serait une Idée esthétique plus qu'un concept d'exposition. Jean-Louis Déotte reformule en ce sens la notion kantienne d'Idée esthétique dans ce contexte de la collection muséale où des parentés inaperçues forment le principe de coordination en fonction du goût et non des anciennes destinations des éléments présentés :

«Le sens de l'Esthétique de Kant serait donc, non un concept par rapport auquel

l'imagination pourrait être adéquate en apportant la représentation nécessaire à

l'exposition dudit concept, mais une Idée esthétique : celle de la Galerie. » ${ }^{7}$

Le jugement porté par le spectateur sur l'exposition n'est pas un jugement déterminant permettant une connaissance, c'est-à-dire fondé sur la reconnaissance d'exemples ou d'expérimentations d'une loi ordonnatrice a priori. Le rapport à la collection exposée ne se fait pas en fonction d'un concept logique qui contraindrait a priori les relations entre les éléments présentés comme des exemples d'une règle déjà connue, mais en fonction de ce qui se révèle dans la somnolence de son parcours et des relations imprévisibles qui émergent. Il s'agit d'un jugement réflexif où il n'existe pas de loi connue ni rien à expérimenter, mais où un principe d'organisation est tiré a posteriori, comme généré, plutôt qu'exemplifié, par ses exemples. Il s'agit ainsi de partir de l'objet exposé et non d'un concept donné pour produire une Idée esthétique plutôt que pour retrouver un concept à partir de ses exemplifications. L'Idée esthétique pourrait dans cette perspective être considérée comme le «principe du glissement des formes les unes sur les autres $»^{8}$, de coordination ou plutôt de circulation entre les éléments d'une exposition.

18 Ainsi, en tant que principe d'un Musée imaginaire qui n'est pas d'abord donné et qui serait à réaliser ou à exemplifier, l'Idée esthétique donne à penser la relation entre des éléments d'une même famille constituée au hasard des rencontres avec les œuvres. L'art des femmes serait alors, la loi de correspondance de toutes ces œuvres exposées, loi déduite de la circulation entre des exemples. La reconnaissance de l'art des femmes 
a ainsi bien lieu dans un jugement réfléchissant, plus qu'il n'est déterminé a priori comme concept de l'exposition : «C'est de la rencontre entre le public et les œuvres dans les salles et de leur réaction que naîtra la réponse $»^{9}$. En tant que tel, l'art des femmes est donc d'un principe de coordination parmi d'autres traversant la série des œuvres constituée par l'art universel. Il est sur le même plan que n'importe quel autre principe présidant à la constitution d'un Musée imaginaire. Dès lors, les femmes semblent bien être devenues des artistes: leurs œuvres sont disponibles de la même manière que celle des artistes masculins et, si dans elles, il n'y a que leurs œuvres, ce choix est du même ordre, et n'est pas plus discriminant, que celui de faire une exposition sur un artiste particulier ou sur n'importe quel thème. Que le nom propre imposé à la collection de chaque visiteur soit non seulement elles, c'est-à-dire l'art féminin, mais également centre pompidou insiste sur cette dimension: il s'agit d'un accrochage comme les autres dans un musée dont le fond permet une telle rotation. Les œuvres des femmes sont au musée comme n'importe quelles œuvres. Le visiteur découvre à travers l'exposition qu'il y a un art des femmes qu'il constitue comme idée du musée imaginaire. Si le rapport à l'exposition est ainsi réflexif, le féminisme qui fut le concept que certaines de ces œuvres ont exemplifié ne saurait apparaître.

\section{L'inutilité du féminisme}

\section{La destination féministe des œuvres exposées}

19 Si le rapport à l'œuvre exposée est similaire, il y a cependant une différence fondamentale entre des statues que voit Picasso et les œuvres de elles. Contrairement à la majeure partie des œuvres contemporaines, un certain nombre de pièces qui y sont présentées on eu une utilité du même ordre que celle des statues de Dieux que voit Picasso, mais cette utilité est encore d'actualité, contemporaine et encore vécue comme telle. Avant d'être simplement d'art, un certain nombre des œuvres exposées dans elles ont été en effet faites pour avoir une influence sur les représentations des femmes. Elles ont été créées et reçues dans cette optique utilitaire. Ces œuvres peuvent être dites en ce sens féministes. Cette qualification ne dépend pas du fait qu'elles soient œuvres de femmes mais qu'elles trouvent un sens dans un certain contexte culturel, social et politique. Pour les féministes, l'art est en effet le lieu de l'activité politique et sociale. Il s'agit de modifier la représentation des femmes, de la décadrer. Elles ont donc mis l'accent sur ce qui se situe hors du cadre de la logique, de la représentation ou de l'histoire pour rendre sensible et faire apparaître d'autres manières de les concevoir, dans lesquelles les femmes auraient conscience de leur situation, voire une place différente. Le texte de Hanna Wilke mis, dans le catalogue, en relation avec son œuvre SOS Starification Object Series : An Adult Game of Mastication, explicite bien cet enjeu :

"Je me suis donné la forme d'une déesse, d'un ange pour pouvoir expulser les symboles féminins créés par les hommes et pour donner ainsi aux femmes un nouveau statut, un nouveau langage formel. [...] Les femmes ont toujours servi d'idéal et d'inspiration créatrice aux hommes. Créer mes propres images en tant qu'artiste et objet était important pour moi, car je refusais totalement d'être l'objet. Je me suis transformée en objet pour idéaliser la femme, à la manière dont les hommes l'ont fait si souvent, pour lui restituer son corps. J'ai repris possession de mon corps au lieu de le faire "créer" par quelqu'un d'autre. ${ }^{10}$ 
L'œuvre a donc un sens étroitement attaché à son contexte. Elle a une fonction non seulement pour l'artiste, mais essentiellement pour le public qui la voit. Il s'agit d'un côté de montrer les normes et les institutionnalisations imposées aux femmes et, en même temps, de faire en sorte que cette prise de conscience agisse comme un appel à une résistance et à la conception de nouvelles possibilités d'existences. La féministe Maria Rosler place explicitement son œuvre dans une telle perspective quand elle écrit, dans un texte repris dans le catalogue de l'exposition pour illustrer son œuvre Semiotics of the Kitchen, qu'elle a vu « cet enregistrement agir comme une libération devant des publics de femmes $»^{11}$. Une telle œuvre intéresse donc son public en participant de la conscience qu'il a de lui même et de son environnement social et culturel. S'il est possible d'en tirer un plaisir purement esthétique devant ses formes ou ses textures, elle n'en a pas moins une utilité attachée au contexte du combat féministe : elle est une arme dans ce combat et est reçue de cette manière.

21 Si des artistes comme Hanna Wilke, Maria Rosler, Nan Goldin (Heartbeat) ou Zoe Léonard (Preserved Head of a Bearded Woman, Musé Orfila) s'inscrivent ainsi explicitement dans une remise en cause des identités sexuelles, même des œuvres qui ne sont pas crées pour avoir cette fonction féministe, comme par exemple celle d'Abramovich (Freeing the Body) ou d'Orlan qui refuse une lecture univoquement féministe, n'interrogent pas moins la place dans la société et les modes de représentation et d'existence des femmes et de leurs corps. Ne serait-ce que parce que la performance fait de leur corps leur œuvre qui existe comme référent par sa propre puissance performative et non plus seulement dans le fantasme masculin. De la même manière, les œuvres d'artistes moins, voire pas du tout, engagées dans le combat féministe, peuvent intéresser un public féministe par leur interrogation sur les stéréotypes féminins (Véronique Ellena, Promenade dans les Calanques de la série les Dimanches). De telles œuvres sont perçues comme des interrogations du champ au sein duquel le genre opère en mettant en action le corps féminin et ses représentations, et sont ainsi vécues par le public de manière intéressée. Ce qui compte pour ces œuvres, c'est non seulement leur matière, mais essentiellement la signification qu'elles prennent, leur sens dans le combat féministe. Dans cette perspective, une très grande partie des œuvres présentées dans elles peuvent être considérées comme féministes, comme intéressant les féministes et donc un contexte social où le féminisme est actif et prend sens. Ne pas les montrer comme telles est un choix d'exposition. Jean-Louis Déotte insiste bien sur le fait qu'un tel choix est celui de l'organisateur de l'exposition ${ }^{12}$ en montrant que, pour Kant, selon la manière dont une chose est vue, elle peut être l'objet d'un jugement réfléchissant, esthétique, ou déterminant, qu'il s'agisse d'une connaissance ou de la simple reconnaissance d'une destination qui concerne le visiteur.

\section{L'organisation esthétique de la collection comme concept d'exposition}

Si la contextualisation était donnée, le visiteur pourrait voir l'histoire du féminisme, dans la mesure où une œuvre d'art peut donner à voir, en l'occurrence, l'intérêt d'un combat social. Même si, dans le cadre de l'exposition, le visiteur se laisserait prendre par la beauté de ce qu'il voit, il saurait qu'il y a quelque chose qui pourrait l'intéresser : en sortant il serait ainsi amené à s'intéresser au féminisme, à voir en quoi ce qu'il a vu n'est pas seulement une exposition d'art - même si cela en est aussi une - mais 
également quelque chose qui le concerne socialement, culturellement et politiquement. Il s'agirait du " plaisir de découvrir que là, sur cette question-là, nous aimerions par exemple, apprendre et revenir. Le plaisir pris à savoir que du savoir nous attend. Nous n'en saurons pas plus : il faudrait retourner aux livres. Mais l'essentiel est là : savoir que quelque chose nous attend $»^{13}$.

Au contraire, la recherche du féminisme à l'occasion de elles débouche sur la déception de ne pas parvenir à être intéressé par quelque chose que nous savons encore nous intéresser. Exposées de la même manière que des œuvres religieuses médiévales qui ne sont plus utilisées comme telles dans la mesure où le monde dans lequel elles servaient une croyance est passé, les œuvres du combat féministe semblent ne plus pouvoir nous être utiles comme si leur monde également était passé, c'est-à-dire comme si le contexte dans lequel le féminisme a un sens social et culturel était passé. Même les quelques évocations elliptiques et non circonstanciées, qui ressemblent plus à des positions philosophiques abstraites qu'à un combat social, vont dans ce sens. Le visiteur fait l'expérience d'œuvres qui témoignent d'un contexte inatteignable et ne se sent pas plus concerné par le féminisme ainsi exprimé qu'il ne le serait par les esprits que rappellent des masques exotiques ou par l'ordonnancement du monde que rappelle les peintures religieuses médiévales.

Même dans le cas où une telle image religieuse médiévale ou un tel masque exotique serait exposé dans un musée d'histoire comme témoignage, son contexte pourrait bien être reconstitué, cet objet-image ne nous sera jamais utile comme aux croyants à son époque et nous seront dans l'incapacité constitutive de le vivre ainsi. Il s'agit d'un objet d'occasion dont la finalité ne peut être reconstituée. Jean-Louis Déotte considère que même une remise en contexte ne peut nous la redonner dans la mesure où on ne peut en faire, à proprement parler, l'expérience : on peut savoir que ces objets ont eu une destination, on peut même éventuellement avoir une idée de cette destination, mais cet objet ne saurait l'exemplifier dans la mesure où le lien entre les deux nous échappe. Leur monde n'est pas le nôtre. En effet, leur finalité relève d'un autre cadre d'expériences que celui qui nous est accessible. En tant que technologie de suspension, le musée établit ainsi d'une discontinuité entre le monde des éléments présentés et celui du visiteur. En effet, là où il n'y a pas discontinuité, les choses présentées intéressent directement, sont encore utiles. Ne subsiste que les restes de ce qui n'a plus d'identité, des choses dont on ignore tout.

«Une telle destination ne peut pas être exposée en réponse à une question de savoir. La "croyance" ne peut répondre à la question que lui pose la science. Ce sont des mondes incompossibles. Étrangeté renforcée par le fait que les "sauvages" privilégiaient une définition de la surface d'inscription générale, qui n'est plus la nôtre : écrivant sur le sol de la Terre, sur les corps, sur les ustensiles, quasi indifféremment. D'ailleurs, nous pourrions dire autant pour les objets du culte chrétien. ${ }^{14}$.

Il en va de même des œuvres du féminisme exposées dans elles. Ce n'est pas que nous sommes incapables de considérer qu'elles ont eu une finalité, nous sommes incapables de l'y intégrer, de la reconstituer. Cette incapacité n'est pas une insuffisance de savoir qui se comblera, dans ce cas il y aurait un concept qu'on ne parviendrait pas à exemplifier de manière satisfaisante. Le visiteur n'en a pas le concept. Le visiteur ne peut comprendre ou ressentir ce à quoi ces œuvres ont été destinées; il peut bien savoir qu'elles ont eu une destination appelée féminisme, mais tout se passe comme si celle-ci nous échappait constitutivement. On ne peut y accéder comme exemplifications 
du concept de féminisme puisque celui-ci nous est inaccessible. Les œuvres présentées ne sauraient trouver de concept dont elles seraient l'exemplification. En termes kantiens, il serait alors possible de dire que le féminisme serait plus de l'ordre de l'Idée que de celui du concept, dans la mesure où il dépasserait les possibilités de l'entendement. Cette Idée serait de plus du côté de l'Idée esthétique, plutôt que de celui de l'Idée de la raison, car ce qui échappe à l'entendement, ce n'est pas un concept trop riche pour être saisi, mais qui n'existe pas moins ; c'est la matière présentée, ce qui est perçu par le visiteur lors de l'exposition, qui ne parvient pas à être subsumé sous un concept qui serait donné a priori. Il ne s'agit pas d'un concept d'exposition inexemplifiable, mais d'une matière d'exposition incompréhensible.

Dans cette mesure, ayant perdu toute destination hors de lui-même, actuellement l'art féministe apparaît comme un objet d'occasion. Les œuvres féministes sont ainsi privées de toute actualité de la même manière que l'est une image religieuse médiévale devant qui plus personne ne s'agenouille au musée. C'est la même image mais elle ne nous affecte plus ou nous affecte esthétiquement. Elle n'a plus sur nous l'impact pour lequel elle a été réalisée. Il reste une œuvre d'art qu'il est impossible de retrouver comme œuvre socialement utile. Le fait d'avoir uniquement un rapport esthétique à ces œuvres signifie donc que le féminisme ne nous concerne plus. Le féminisme« est destitué en même temps de son importance dans le monde [...] [reste] un double muséal - une ruine - pour une œuvre qui en son temps a eu une fonction $»^{15}$.

En effet, Jean-Louis Déotte montre que mettre une œuvre qui a eu une utilité sociale, culturelle ou politique au musée signifie prendre acte de la fin de cette utilité. À le suivre, l'institution du musée en France par la Révolution consiste à établir une distance incommensurable avec le contexte social et culturel de ce qui est exposé. Une distance avec l'ancien monde est produite en exhibant ses objets devenus d'occasion, car «[le Musée] est toujours Musée de la loi défunte $»^{16}$. Il s'agit alors d'intégrer ces objets à l'histoire de l'art universelle comme ceux d'une époque révolue de l'humanité aux côtés des ruines de toutes les destinations singulières subsumées sous un regard. Cette suspension semble être la condition de la volonté de elles d'inclure les femmes dans une perspective universalisant au delà de leur place sociale et culturelle particulière. Les œuvres du féminisme sont ainsi accueillies dans l'art universel, aux côtés de celles du christianisme, de la monarchie et de toutes ces œuvres dont l'utilité n'est plus.

Mettre ces œuvres féministes au musée, c'est non seulement d'une certaine manière neutraliser cette puissance de destination encore active en faisant comme si elle était passée, mais c'est également entériner cette mort en faisant en sorte que ce qui faisait la spécificité active de ces œuvres ne soit plus pertinent pour définir la relation que le public a à elles. Cette perte est ce que donne à voir la pièce de Jana Sterbak, Vanitas. Robe de chair pour albinos anorexique: "Vanitas pourrait également évoquer les changements que le temps imprime à la perception des œuvres. Le jour du vernissage, quand on expose la robe, la chair est crue. Puis la viande sèche et commence à ressembler au cuir; elle devient alors acceptable. Cela est aussi vrai des artistes. Certains conservateurs préfèrent travailler avec des artistes morts, car ils dérangent moins $»^{17}$. Le féminisme ne serait plus ce qui peut interpeller, mais simplement ce qui a produit de belles œuvres.

29 C'est ce que signifie la volonté de montrer que le critère de genre, plus que de n'être pas pertinent, n'est pas opératoire pour représenter l'art du xxe siècle: «Que cette 
représentation des femmes et/ou des hommes n'ait au final plus d'importance est l'objectif $\aleph^{18}$. Cette remarque montre assez que c'est là le concept déterminant de l'exposition. C'est ce que montre elles, ce que l'exposition de chaque œuvre ou leurs mises en relation exemplifient. Le concept qu'elles exemplifie en faisant que les œuvres soient perçues sans concept à exemplifier, c'est que le féminisme ne nous concerne plus, qu'il est d'une autre époque. La collection serait alors organisée selon un concept a priori, même s'il n'en à pas l'air: le désintérêt pour le féminisme, le fait que le féminisme soit du côté de l'Idée esthétique plutôt que du concept, serait son principe d'organisation. Le visiteur apprend que le féminisme est un objet d'occasion et c'est ce savoir qui lui permet de les goûter esthétiquement. En exposant les œuvres féministes de manière esthétique, il s'agit donc d'exemplifier le fait que le féminisme ne nous concerne plus, de la même manière que la Révolution française a exposé les œuvres de l'Ancien Régime pour « faire sentir que gît là, dans ces objets, un autre temps une autre époque de la destination [...] faire sentir en quoi notre époque s'éloigne substantiellement de ces époques et de ces destinations marquées par l'altérité et en quoi, même si le passé (ou l'exogène) n'est pas totalement mort, notre temps leur est irrévocablement étranger parce qu'il est étranger à lui-même $»^{19}$. La puissance sociale, politique et culturelle du féminisme est ainsi neutralisée par sa patrimonialisation que constitue la prise en charge de ses œuvres par le musée elles.

\section{Peut-il y avoir de grands artistes féministes ?}

\section{L'exposition féministe des femmes artistes}

30 Tout se passe comme si le rapport du visiteur de l'exposition à l'utilité de ces œuvres était le même que celui de Picasso qui ne se sent pas très concerné par l'utilisation religieuse des statues des Cyclades. Que se soit en suivant un intérêt esthétique ou un intérêt culturel et historique, cette utilisation ne le concerne pas : voir dans les œuvres présentées dans elles que des œuvres d'art suggèrent que leur utilité ne concerne pas le visiteur d'une exposition, qui pourtant aurait été sûrement impossible sans les transformations que ces œuvres ont induites. Ainsi, les œuvres exposées ne le sont pas comme des œuvres féministes, c'est à dire utiles à la modification de la perception sociale et culturelle des femmes. Dans la perspective de elles, se serait exactement cela qui serait une pratique féministe : dans la mesure où n'étant plus féministes mais d'art, le genre des artistes exposées ne serait plus pertinent dans la compréhension de leurs œuvres. C'est ainsi qu'est définit l'enjeu politique de elles en tant que rien de moins que «l'endroit où le monde se pense radical, utopique, extrême, provocateur ${ }^{20}$. Cet enjeu politique ne se réalise pas directement par la mise en avant activiste des enjeux du féminisme, mais curieusement par leur suspension, la décision de ne pas les prendre en considération.

31 Paradoxalement donc, au niveau interne muséographique, l'exposition montre que le féminisme des œuvres exposées nous concerne à peu près autant que la religion des Cyclades concernait Picasso, mais, au niveau externe social, elle aurait une utilité finalement féministe comme si celui-ci concernait encore la société. Le paradoxe se redouble si l'on considère comme Kant, qu'une galerie d'art est elle-même une œuvre d'art. Si c'est bien le cas, alors ce qui, dans cette exposition, est utilitaire devrait être également suspendu pour n'être qu'art. Une solution serait de considérer que les 
œuvres sont simplement d'art et doivent être goûtées sans que leur utilité ne parasite la sensation esthétique, alors qu'une exposition est essentiellement utilitaire. L'antinomie serait la suivante: un geste féministe qui ne peut avoir lieu que si le féminisme n'est plus pertinent pour comprendre ce qu'il expose. Cependant, d'une certaine manière ces œuvres ont rendu possible elles en influant directement sur les perceptions et représentations des femmes. Si considérer que les œuvres des femmes sont des œuvres d'art est, comme l'affirme la commissaire de l'exposition, un "geste féministe ${ }^{21}$ qui cherche à modifier la place des femmes dans les rapports sociaux en leur donnant accès aux conditions matérielles nécessaires pour occuper une place jusque là réservée aux hommes, alors elles devrait s'inscrire dans la continuité utilitaire des œuvres qu'elle présente.

Il semble que, pour la commissaire de l'exposition, la disqualification de la pertinence du critère de genre soit ainsi une démarche plus féministe que le combat pour la reconnaissance d'une place, peut-être égale en droit, mais non moins spécifique des femmes dans la société en général et dans le milieu de l'art en particulier. Le féminisme ne passerait donc pas par une transformation, mais par un nivellement social.

Ce faisant, et malgré la citation de son célèbre article, elles ne répond pas à la question que pose Lindan Nochlin. Elle la déplace. Dans la perspective de elles, la possibilité de reconnaissance des femmes comme grands artistes passe par la négation des conditions sociales définissant la place particulière des femmes dans les dispositifs de production, de reconnaissance et d'existence artistiques. Elles traite plus de l'art que des femmes, et c'est d'ailleurs son ambition affichée. L'exposition semble ainsi répondre à la question : «Pourquoi il n'y a pas de femmes grands artistes?». Cette question porte alors sur la possibilité pour des femmes de devenir des grands artistes. L'enjeu est la possibilité pour des femmes d'être subsumées dans la catégorie universelle du grand artiste. L'accent est mis sur les œuvres et les conditions d'exposition plus que sur les artistes en prise avec leur condition sociale.

La question de Lindan Nochlin est plutôt: "Pourquoi il n'y a pas de grands artistes femmes? ». Il ne s'agit pas de demander s'il est possible que des grands artistes soient des femmes, mais s'il est possible qu'ils soient de grands artistes en tant que femmes. La question met en avant le genre des artistes. Dans cette perspective, qui est celle par exemple de l'exposition Global Feminism, l'enjeu politique est alors la différenciation de la catégorie universelle grand artiste en masculin et en féminin. Il ne s'agit pas ici de refaire un commentaire de l'article de Lindan Nochlin, mais simplement de remarquer qu'elle pose une question qui concerne les femmes, plus que l'art, c'est-à-dire les conditions sociales propres aux femmes.

\section{Le féminisme comme politique de l'art}

Il s'agit ainsi non seulement d'un choix d'exposition, mais essentiellement d'un choix à la fois stratégique et politique, et en fait également ontologique : le féminisme est-il la reconnaissance que les femmes sont comme des hommes comme les autres, dans une même humanité au delà des déterminations sociales et culturelles; ou bien est-ce un combat pour l'existence sociale des femmes en tant qu'elles sont le produit de la différentiation sociale et culturelle des genres? essentialisme universaliste s'intégrant parfaitement dans le contexte idéologique 
français de l'intégration républicaine, dans la mesure où il s'agit de montrer, comme veulent le faire également par exemple les lois sur la parité, que le critère du genre n'est pas pertinent. Il empêcherait en fait de voire l'égalité fondamentale entre les hommes et les femmes. Le féminisme serait donc la suppression du contexte social déterminant les différences de genre pour découvrir que les femmes sont des hommes comme les autres. Il s'agit de revenir à une sorte d'égalité qui serait naturelle dans la mesure où elle est constatative : on s'est aperçu qu'il y avait suffisamment d'œuvres de femmes, donc virtuellement autant que d'œuvres d'hommes - ce qui ne signifie pas le même nombre - dans les collections d'un musée pour constituer l'accrochage de l'exposition permanente. Pour faire cet accrochage, il suffisait donc de supprimer le critère du genre, c'est-à-dire les déterminations sociales et culturelles pour en revenir à ce qui avait toujours été là, mais caché : dans ce que l'art a produit au $\mathrm{xx}^{\mathrm{e}}$ siècle, les œuvres des femmes ont une importance quantitative. Si femmes et hommes ont ainsi produit suffisamment d'œuvres pour faire un accrochage au centre Pompidou, c'est bien qu'ils sont égaux. Il s'agit donc à peine d'une politique. L'opposition à la rigidité des déterminations sociales de genre produit exactement le contraire de l'essentialisme différentialiste des féministes françaises des années 1970 qui cherchaient certes à retrouver ce que les déterminations sociales de genres avaient caché mais pour y découvrir une essence féminine propre.

Si cette conception du féminisme apparaît dans l'espace public de manière privilégiée à l'occasion de elles, c'est qu'elle reproduit d'une certaine manière la même logique que celle de l'esthétique au sens où Kant la définit dans la Critique de la faculté de juger. D'un côté l'intérêt, c'est-à-dire les déterminations sociales et culturelles que les hommes peuvent trouver aux choses de la nature ou de l'art, est suspendu pour que la beauté puisse être constatée de manière universelle ; d'un autre coté, le féminisme suspend les déterminations sociales et culturelles pour permettre de constater l'égalité des hommes et des femmes. Se sont bien en effet ces deux mouvements parallèles qui fonctionnent dans elles. Dans ce cadre, si les femmes peuvent être de grands artistes comme les autres, ce n'est pas le cas des féministes qui revendiquent une transformation des cadres sociaux et culturels qui déterminent l'existence des femmes. Il ne peut y avoir d'art féministe que si cet art s'accompagne du désintérêt pour le combat des femmes. Sinon, art féministe serait un double oxymore. D'un point de vue esthétique, considérer une œuvre comme féministe signifierait reconnaître son utilité dans un contexte social et culturel qui est encore le nôtre : elle intéresserait, donc ne pourrait être esthétiquement goûté. D'un point de vue social, cela signifierait que le genre de l'artiste n'est pas indifférent : reprenant à son compte les déterminations de genre, une telle œuvre ne serait pas féministe, au sens universaliste du terme.

À ce féminisme s'oppose une autre conception : celle d'un combat pour que les femmes aient une place sociale aussi viable et vivable que celle des hommes, sans pourtant être la même. Si l'œuvre par excellence du féminisme universaliste est l'exposition elles, les œuvres qui s'y trouvent sont le produit de cet autre féminisme, disons politique au sens où il active des transformations sociales. Ces œuvres sont dans ce cadre indiscutablement utilitaires, elles nous intéressent socialement, culturellement et politiquement. Elles transforment notre rapport aux femmes. Comment alors comprendre qu'il puisse s'agir d'œuvres d'art?

Si, pour reprendre la formule de Norbert Elias, l'art fut utilitaire avant de devenir l'art, dire qu'il y ait des œuvres d'art en ce sens féministes serait encore une fois un 
oxymore. Il faudrait en effet que l'art puisse être à la fois utilitaire et en même temps simplement l'art. La conclusion pourrait être qu'il faut choisir, et alors, le centre Pompidou étant un musée d'art et non un musée d'histoire, elles serait le seul choix d'exposition possible. S'il faut cependant prendre au sérieux la revendication artistique des artistes se disant ou étant reconnues comme féministes, alors il faut penser une troisième époque de l'art où ce qui semble inconciliable fonctionnerait en même temps, et non pas l'un après l'autre dans deux époques ou dans deux expositions différentes. Ce choix oxymorique d'exposition est le défit que pose l'art féministe, à la muséographie, et qui la ferait entrer dans une époque, si l'on veut postkantienne ou postmoderne. C'est la condition d'un art politique, plutôt que d'une politique de l'art.

\section{NOTES}

1. Art News, $n^{\circ} 69$, janvier 1971, repris dans Femmes, art et pouvoir, et autres essais, Éditions Jacqueline Chambon, 1993.

2. Picasso, cité dans Jean-Louis Déotte, Le musée, l'origine de l'esthétique, L'Harmattan, 1993,p. 361.

3. Camille Morineau, Catalogue, p. 14-15.

4. Camille Morineau, préface du Catalogue, p. 12-13.

5. Voir Griselda Pollock, Generations and Visual Arts : Feminist Reading, New-York, Routledge, 1996.

6. George Salles, Le Regard (1939), p. 35, cité dans Jean-Louis Déotte (1993), p. 187-188.

7. Jean-Louis Déotte (1993), p. 236.

8. J. Jamin, cité dans Jean-Louis Déotte (1993), p. 363.

9. Camille Morineau, p. 19.

10. Camille Morineau, p. 67.

11. Camille Morineau, p. 178.

12. Jean-Louis Déotte (1993), p. 129 et 206.

13. Jean-Louis Déotte (1993), p. 338.

14. Jean-Louis Déotte (1993), p. 357.

15. Jean-Louis Déotte, Oubliez ! L'Europe, les ruines, le Musée, L'Harmattan, 1994, p. 36.

16. Jean-Louis Déotte (1994), p. 61.

17. Camille Morineau, p. 94.

18. Camille Morineau, p. 16.

19. Jean-Louis Déotte (1994), p. 220.

20. Camille Morineau, p. 16.

21. Camille Morineau, p. 16. 


\section{AUTEUR}

NATHANAËL WADBLED

doctorant en philosophie (Paris 8), associé au centre d'Étude féminine et d'Étude de Genre 